\title{
Editorial: Gut Health: The New Paradigm in Food Animal Production
}

\author{
Michael H. Kogut ${ }^{1 *}$ and Ryan J. Arsenault ${ }^{2}$ \\ ${ }^{1}$ USDA Agricultural Research Service, College Station, TX, USA, ${ }^{2}$ University of Delaware, Newark, DE, USA
}

Keywords: gut health, production animals, Chickens, Swine, Cattle, microbiome, mucosal immunity

\section{The Editorial on the Research Topic}

Gut Health: The New Paradigm in Animal Production

Optimal gut health is of vital importance to the performance of production animals. Gut health is synonymous in animal production industries with animal health. Although there does appear to be a direct relationship between animal performance and a "healthy" gastrointestinal tract (GIT), there is no clear definition for "gut health" that encompasses a number of physiological and functional features, including nutrient digestion and absorption, host metabolism and energy generation, a stable microbiome, mucus layer development, barrier function, and mucosal immune responses (1-8). The GIT is responsible for regulating physiological homeostasis that provides the host the ability to withstand infectious and non-infectious stressors (9-19). Understanding the interactions between these diverse physiological features emphasizes the extent of areas encompassed by gut health and the ability to regulate animal production. For our part, we will define gut health as the absence/ prevention/avoidance of disease so that the animal is able to perform its physiological functions in order to withstand exogenous and endogenous stressors. Furthermore, worldwide public concerns about the production animal industries' dependency on the use of growth-promoting antibiotics (AGPs) have resulted in the ban of AGPs by the European Union and a reassessment of their use in the United States. Thus, current research is focused on alternatives to antibiotics for sustainable food animal production (20).

A recent Research Topic in Frontiers in Veterinary Infectious Diseases was on gut health and wondering whether we should consider gut health as the new standard when considering animal production. The objective of this Editorial is not to review the literature on gut health in production animals, but, rather, it is our attempt to summarize findings of the 15 papers that were published within this Research Topic. Obviously, the Topic was not comprehensive in the production animal commodity reported, but it was a very good overview of the current status of the ongoing work in gut health and physiology within the veterinary community.

\section{GUT MICROBIOME}

The complex gut microbiome is not a silent organ or a collection of passenger microorganisms; but rather, the intestinal microbial community represents active participants in vertebrate immunity and physiology. The gut microbiota confers health benefits to the host, including aiding in the digestion and absorption of nutrients, contributing to the construction of the intestinal epithelial barrier, the development and function of the host immune system, and competing with pathogenic microbes to prevent their harmful propagation $(18,21)$. Unlike the host genome, which is rarely manipulated by xenobiotic intervention, the microbiome is readily changeable by diet, ingestion of antibiotics, infection by pathogens, and other life events [Danzeisen et al.; Ballou et al.; Mon et al.; Malmuthauge et al.; (8)]. 
Antibiotics have a great effect on the host normal microbiota upsetting the balance and inducing a dysbiotic state (8). The use of sub-therapeutic doses of antibiotics in animal diets have been a common practice for promoting growth due to their ability to increase feed efficiency or preventing diseases. Danzeisen et al. used a sub-therapeutic concentration of penicillin to define beneficial members of the microbiome in turkeys that resulted in increased feed efficiency and enhanced growth. By identifying the specific bacterial populations responsible for improved performance, the authors hypothesize that these bacteria can then be used as probiotics.

The microbiome has a direct effect on the development and function of the mucosal immune system. Malmuthauge et al. found significant associations between the microbiome and the expression of genes regulating the mucosal barrier and innate immunity in neonatal cattle. Regional differences in the microbiome were associated with regional differences in innate immune gene transcription. Similar findings were described between the microbiome of broiler chickens and the expression of avian cytokine RNA transcripts (Oakley and Kogut). A negative correlation between pro-inflammatory cytokine genes and the phylum Firmicutes was found; whereas a positive correlation was identified with the pro-inflammatory cytokines and the phylum Proteobacteria.

Wigley and Ballou et al. asked the questions: what constitutes a normal or healthy microbiome and what effects do treatments that are being used to improve gut health (vaccines and probiotics) have on the development of the gut microbiome? Wigley pointed out that certain bacteria, such as Escherichia coli, Clostridium perfringens, and Campylobacter, are often considered commensals and part of the cecal microbiome. The removal of AGPs, manipulation of the cecal microbiome, changing husbandry practices, and other internal and external factors lead to changes in the host responses that result in "new" infections (22-25). Using a live attenuated Salmonella vaccine or a lactic acid bacteria probiotic, Ballou et al. characterized the effects of gut health treatments have on the microbiome. Alterations in microbial diversity in the microbiome of young chicks given the vaccine and, to a less extent with the probiotic, were found, which were independent of bacterial colonization by the treatments. The microbiome alterations were maintained through 28 days of age, suggesting that early exposure to certain bacteria may permanently influence the microbial diversity in the microbiomes. Similar results were described by Mon et al. where a Salmonella infection in day-old chicks induced a profound decrease in microbial diversity in the cecum. Specifically, there was an increase in Enterobacteriaceae and a decrease in butyrate-producing bacteria in the Lachnospiraceae family implying that exposure to a Salmonella infection early after hatch can impact the composition of the developing microbiome that affects colonization resistance to microbial pathogens.

Yeast-derived dietary supplements are increasingly being used as pre- and probiotics to improve gut health (26). Roto et al. detailed the effects of yeast-derived compounds in livestock diets and their effect of the microbiome. The use of yeast-derived compounds as supplements in livestock diets improved performance, increased beneficial bacteria in the microbiome, and increased immune responsiveness. Additionally, the yeast-derived products are cost-effective, do not induce antimicrobial resistance in pathogens, and, because of their multiple mechanisms of action, can be used in the variety of environments found in livestock industries.

\section{MUCOSAL IMMUNE RESPONSE}

The intestinal tract is an active immunological organ with more resident immune cells than anywhere else in the body. They are organized in lymphoid structures called Peyer's patches and isolated lymphoid follicles, such as the cecal tonsils. Macrophages, dendritic cells, various subsets of T cells, B cells, and secretory IgA all contribute to the generation of a proper immune response to invading pathogens, while keeping the resident microbial community in check without generating an overt inflammatory response.

In addition to the immune cells, the intestinal epithelial cells contribute to mucosal immunity (21). A single layer of epithelial cells separates the densely colonized and environmentally exposed intestinal lumen from the sterile subepithelial tissue, maintains homeostasis in the presence of the enteric microbiota, and contributes to rapid and efficient antimicrobial host defense in the event of infection with pathogens. Both epithelial antimicrobial host defense and homeostasis rely on signaling pathways induced by innate immune receptors demonstrating the active role of epithelial cells in the host-microbial interplay. Lastly, a layer of mucus overlying the intestinal epithelium forms a physical barrier between the mucosa and the resident microbiota, minimizing both microbial translocation and excessive immune activation by the resident microbes.

Intestinal integrity is fundamentally important for the growth and performance of food animals. One of the main advantages of AGPs in animal feed was the reduction in the low-grade, food-induced chronic inflammation that would otherwise be detrimental to animal growth (27). Removal of AGPs from animal feeds results in an increase in enteric disorders, infections, and diseases $(24,25,28,29)$. One of the issues with determining dysfunction of the gut barrier is the lack of specific biomarkers. Two papers in the Research Topic described new methods that: (a) identify serum and tissue biomarkers of gut barrier function (Chen et al.) and (b) identify a non-invasive means to measure gut inflammation as a marker of gut leakage (Kuttappan et al.). Additionally, Ayoola et al. found that the addition of supplemental enzymes ( $\beta$-mannanase, a blend of xylanase, amylase, protease) to the diet of turkeys reduced food-induced inflammation.

One of the main immune functions of the epithelial cell surface is the production of antimicrobial peptides or host defense peptides [HDPs; Ref. (30)]. HDPs are a diverse group of small molecules that possess antimicrobial, immunomodulatory, and barrier function enhancing activities. Robinson et al. described several classes of small-molecule compounds that induce specific induction of endogenous HDP. Furthermore, supplementation of these HDP-inducing compounds enhanced bacterial clearance, improved enteric barrier integrity, and improved animal production efficiency with minimal intestinal inflammation.

The host/pathogen interactome leads to a number of immune and biochemical changes at the infection site as the 
pathogen tries to derive nutrients from the host, while the host uses immunometabolic countermeasures against the pathogen. Arsenault and Kogut developed a novel tool that characterizes the immunometabolic phenotype of infected cells/tissues. The kinome peptide array identifies alterations in phosphorylation events in both immunity and metabolism simultaneously. The kinome array was used to identify the immune changes occurring in the cecum of chickens during the establishment of a persistent, asymptomatic Salmonella infection (Kogut and Arsenault). A number of immune signaling pathways were activated at the site of infection that indicates the development of a tolerogenic response allowing the bacteria to establish a persistent infection.

\section{DIRECT FED MICROBIALS}

The increased use of grains as alternative energy sources in poultry diets has led to an issue with higher levels of less digestible carbohydrates that result in an increase in digesta viscosity and food-induced inflammation. One alternative to optimize digestibility of these complex carbohydrates is the inclusion of dietary enzyme supplements. Latorre et al. took this concept a step further and described the selection of a Bacillus spp. direct fed microbial (DFM) candidate based on their capacity to produce enzymes that breakdown these complex carbohydrates. Bacillus spp. that produced cellulose and xylanase were used as DFM and were found to reduce digesta viscosity and reduce $C$. perfringens growth in a number of different diets containing different complex carbohydrates.

\section{REFERENCES}

1. Shakouri MD, Iji PA, Mikkelsen LL, Cowieson AJ. Intestinal function and gut microflora of broiler chickens as influenced by cereal grains and microbial enzyme supplementation. J Anim Physiol Anim Nutr (2009) 93(5):647-58. doi:10.1111/j.1439-0396.2008.00852.x

2. McCracken V, Gaskins H. Probiotics and the immune system. In: Tannock G, editor. Probiotics: A Critical Review. Helsinki: Horizon Scientific Press (1999). p. 85-111.

3. Nurmi E, Nuotio L, Schneitz C. The competitive exclusion concept: development and future. Int J Food Microbiol (1992) 15(3-4):237-40. doi:10.1016/ 0168-1605(92)90054-7

4. Beckmann L, Simon O, Vahjen W. Isolation and identification of mixed linked beta-glucan degrading bacteria in the intestine of broiler chickens and partial characterization of respective 1,3-1,4-beta-glucanase activities. JBasic Microbiol (2006) 46(3):175-85. doi:10.1002/jobm. 200510107

5. Qu A, Brulc JM, Wilson MK, Law BF, Theoret JR, Joens LA, et al. Comparative metagenomics reveals host specific metavirulomes and horizontal gene transfer elements in the chicken cecum microbiome. PLoS One (2008) 3(8):e2945. doi:10.1371/journal.pone.0002945

6. Dunkley KD, Dunkley CS, Njongmeta NL, Callaway TR, Hume ME, Kubena LF, et al. Comparison of in vitro fermentation and molecular microbial profiles of high-fiber feed substrates incubated with chicken cecal inocula. Poult Sci (2007) 86(5):801-10. doi:10.1093/ps/86.5.801

7. van Der Wielen PW, Biesterveld S, Notermans S, Hofstra H, Urlings BA, van Knapen F. Role of volatile fatty acids in development of the cecal microflora in broiler chickens during growth. Appl Environ Microbiol (2000) 66(6):2536-40. doi:10.1128/AEM.66.6.2536-2540.2000

8. Oakley BB, Lillehoj HS, Kogut MH, Kim WK, Maurer JJ, Pedroso A, et al. The chicken gastrointestinal microbiome. FEMS Microbiol Lett (2014) 360(2):100-12. doi:10.1111/1574-6968.12608
A group of natural products known as phytobiotics have been the focus of several studies in recent years as antibiotic alternatives (31). Phytobiotics are plant-derived products used in feed that possess antimicrobial activity, provide antioxidative effects, enhance palatability, improve gut functions, and promote growth. Murugesan et al. compared the effects of a commercial phytogenic feed additive on growth, intestinal morphology, and microbial composition in chickens to the effects of an AGP. Improved growth, increased intestinal villus height, and decreased total cecal numbers of Clostridium and anaerobic bacteria were comparable between the two treatments. However, birds fed the phytobiotic additives had a significant reduction in coliforms and an increase in Lactobacillus spp. implying an environment that was more suitable for the establishment of growth-promoting bacteria in the microbiome.

Although the GIT is frequently described simply as "the gut," it is actually made up of (1) an epithelium; (2) a diverse and robust immune arm, which contains most of the immune cells in the body; and (3) the commensal bacteria, which contain more cells than are present in the entire host organism. Understanding of the crosstalk between ALL of these interrelated components of the gut is what cumulatively makes the gut the basis for the health of animals and the motor that drives their performance.

\section{AUTHOR CONTRIBUTIONS}

All authors listed have made substantial, direct, and intellectual contribution to the work and approved it for publication.

9. Crhanova M, Hradecka H, Faldynova M, Matulova M, Havlickova H, Sisak F, et al. Immune response of chicken gut to natural colonization by gut microflora and to Salmonella enterica serovar enteritidis infection. Infect Immun (2011) 79(7):2755-63. doi:10.1128/IAI.01375-10

10. Arsenault RJ, Kogut MH. Salmonella enterica Typhimurium infection causes metabolic changes in the chicken muscle involving AMPK, fatty acid and insulin mTOR signaling. Vet Res (2013) 44:35. doi:10.1186/1297-9716-44-35

11. Sansonetti PJ. War and peace at the mucosal surface. Nat Rev Immunol (2004) 4:953-64. doi:10.1038/nri1499

12. Bartlett JR, Smtih MO. Effects of different levels of zinc on the performance and immunocompetence of broilers under heat stress. Poult Sci (2003) 82:1580-8. doi:10.1093/ps/82.10.1580

13. Garriga C, Hunter RR, Amat C, Planas JM, Mitchell MA, Moreto M. Heat stress increases apical glucose transport in the chicken jejunum. Am J Physiol Regul Integr Comp Physiol (2006) 290:195-201. doi:10.1152/ajpregu.00393.2005

14. Hart A, Kamm MA. Mechanisms of initiation and perpetuation of gut inflammation by stress. Aliment Pharmacol Ther (2002) 16:2017-28. doi:10.1046/j.1365-2036.2002.01359.x

15. Quintero-Fiho WM, Ribeiro A, Ferraz-de-Paula V, Pinheiro ML, Sakai M, Sa LRM, et al. Heat stress impairs performance parameters, induces intestinal injury, and decreases macrophage activity in broiler chickens. Poult Sci (2010) 89:1905-14. doi:10.3382/ps.2010-00812

16. Quintero-Fiho WM, Rodrigues MV, Ribeiro A, Ferraz-de-Paula V, Pinheiro ML, Sa LRM, et al. Acute heat stress impairs performance parameters and induces mild intestinal enteritis in broiler chickens: role of acute hypothalamic-pituitary-adrenal axis activation. J Anim Sci (2012) 90:1986-94. doi:10.2527/jas.2011-3949

17. Wideman RF, AL-Rubaye A, Kwan YM, Blankenship J, Lester H, Mitchell KN, et al. Prophylactic administration of a combined prebiotic and probiotic or therapeutic administration of enrofloxacin to reduce the incidence of bacterial chrondronecrosis with osteomyelitis in broilers. Poult Sci (2015) 94:25-36. doi: $10.3382 / \mathrm{ps} /$ peu025 
18. Maslowski KM, Mackay CR. Diet, gut microbiota and immune responses. Nat Immunol (2010) 12:5-19. doi:10.1038/ni0111-5

19. Choct M, Dersjant-Li Y, McLeish J, Persker M. Soy oligosaccharides and soluble non-starch polysaccharides: a review of digestive, nutritive, and anti-nutritive effects in pigs and poultry. Asian-Australas J Anim Sci (2010) 23:1386-98. doi:10.5713/ajas.2010.90222

20. Seal B, Lillehoj HS, Donovan DM, Gay CG. Alternatives to antibiotics: a symposium on the challenges and solutions for animal production. Anim Health Res Rev (2013) 14:78-87. doi:10.1017/S1466252313000030

21. Yu LC-H, Wang J-T, Wei S-C, Ni YS. Host microbial interactions and regulation of intestinal epithelial barrier function: from physiology to pathology. World J Gastrointest Pathophysiol (2012) 15:27-43. doi:10.4291/wjgp.v3.i1.27

22. Stanley D, Geier MS, Hughes RJ, Denman SE, Moore RJ. Highly variable microbiota development in the chicken gastrointestinal tract. PLoS One (2013) 8:e84290. doi:10.1371/journal.pone.0084290

23. Guabiraba R, Schouler C. Avian colibacillosis: still many black holes. FEMS Microbiol Lett (2015) 362:15. doi:10.1093/femsle/fnv118

24. Humphrey S, Chaloner G, Kemmett K, Davidson N, Williams N, Kipar A, et al. Campylobacter jejuni is not merely a commensal in commercial broiler chickens and affects bird welfare. Mbio (2014) 5:e1364-1314. doi:10.1128/ mBio.01364-14

25. Dumas MD, Polson SW, Ritter D, Ravel J, Gelb J Jr, Morgan R, et al. Impacts of poultry house environment on poultry litter bacterial community composition. PLoS One (2011) 6:e24785. doi:10.1371/journal.pone.0024785

26. Sun X, McElroy A, Webb KE, Seftin AE, Novak C. Broiler performance and intestinal alterations when fed drug-free diets. Poult Sci (2005) 84:1294-302. doi:10.1093/ps/84.8.1294
27. Niewald TA. The non-antibiotic anti-inflammatory effect of antimicrobial growth promoters, the real mode of action? A hypothesis. Poult Sci (2007) 86:605-9. doi:10.1093/ps/86.4.605

28. Taira K, Nagai T, Obi T, Takase K. Effect of litter moisture on the development of footpad dermatitis in broiler chickens. J Vet Med Sci (2014) 76:583-6. doi:10.1292/jvms.13-0321

29. Awad WA, Molnár A, Aschenbach JR, Ghareeb K, Khayal B, Hess C, et al. Campylobacter infection in chickens modulates the intestinal epithelial barrier function. Innate Immun (2015) 21:151-60. doi:10.1177/17534259 14521648

30. Brogden KA, Ackermann M, McCray PB Jr, Tack BF. Antimicrobial peptides in animals and their role in host defences. Int J Antimicrob Agents (2003) 22:465-78. doi:10.1016/S0924-8579(03)00180-8

31. Windisch W, Schedle K, Plitzner C, Kroismayr A. Use of phytogenic products as feed additives for swine and poultry. J Anim Sci (2007) 86:E140-8. doi:10.2527/jas.2007-0459

Conflict of Interest Statement: The authors declare that the research was conducted in the absence of any commercial or financial relationships that could be construed as a potential conflict of interest.

Copyright (c) 2016 Kogut and Arsenault. This is an open-access article distributed under the terms of the Creative Commons Attribution License (CC BY). The use, distribution or reproduction in other forums is permitted, provided the original author(s) or licensor are credited and that the original publication in this journal is cited, in accordance with accepted academic practice. No use, distribution or reproduction is permitted which does not comply with these terms. 